$\underline{\text { Supporting Information for }}$

\title{
Ruddlesden-Popper Hybrid Lead Iodide Perovskite 2D Homologous Semiconductors
}

Constantinos C. Stoumpos, ${ }^{1, \ddagger}$ Duyen H. Cao, ${ }^{1, \ddagger}$ Daniel J. Clark, ${ }^{2}$ Joshua Young, ${ }^{3,4}$ James M. Rondinelli, ${ }^{4}$ Joon I. Jang, ${ }^{2}$ Joseph T. Hupp, ${ }^{1}$ and Mercouri G. Kanatzidis ${ }^{1 *}$

${ }^{1}$ Department of Chemistry, Northwestern University, 2145 Sheridan Road, Evanston, Illinois 60208, USA

${ }^{2}$ Department of Physics, Applied Physics and Astronomy, Binghamton University, P.O. Box 6000, Binghamton, New York 13902, USA

${ }^{3}$ Department of Materials Science and Engineering, Drexel University, 3141 Chestnut Street, Philadelphia, Pennsylvania 19102, USA

${ }^{4}$ Department of Materials Science and Engineering, Northwestern University, 2220 Campus Drive, Evanston, IL 60208, USA

\section{Table of contents}

Section S1. Optical Spectroscopy

Section S2. Vibrational spectroscopy

Section S3. Complete crystallographic information

A. Atomic coordinates and anisotropic thermal parameters of $(B A)_{2}(M A)_{n-1} P_{n} \mathrm{I}_{3 n+1} \quad(n=2-4)$

B. Complete crystallographic information for the refinements of $(B A)_{2}(M A)_{n-1} P_{n n} I_{3 n+1}(n=2-4)$ in centrosymmetric space groups 
S1. Optical Spectroscopy

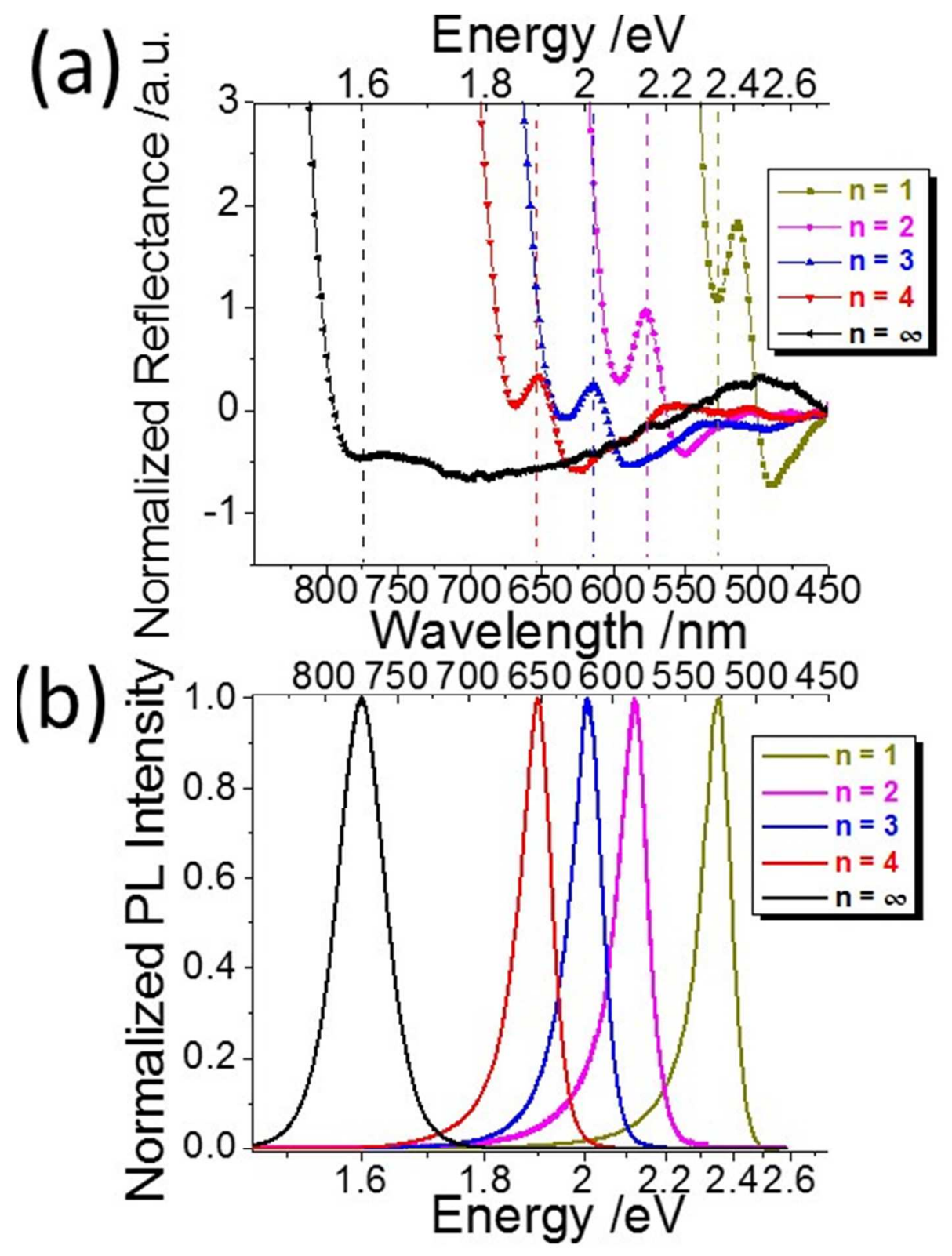

Figure S1. (a) Raw diffuse reflectance data arbitrarily normalized at $450 \mathrm{~nm}$, highlighting the presence of the excitonic peaks. The dashed lines indicate the position of the PL emission plotted in (b). 


\section{S2. Vibrational Spectroscopy}
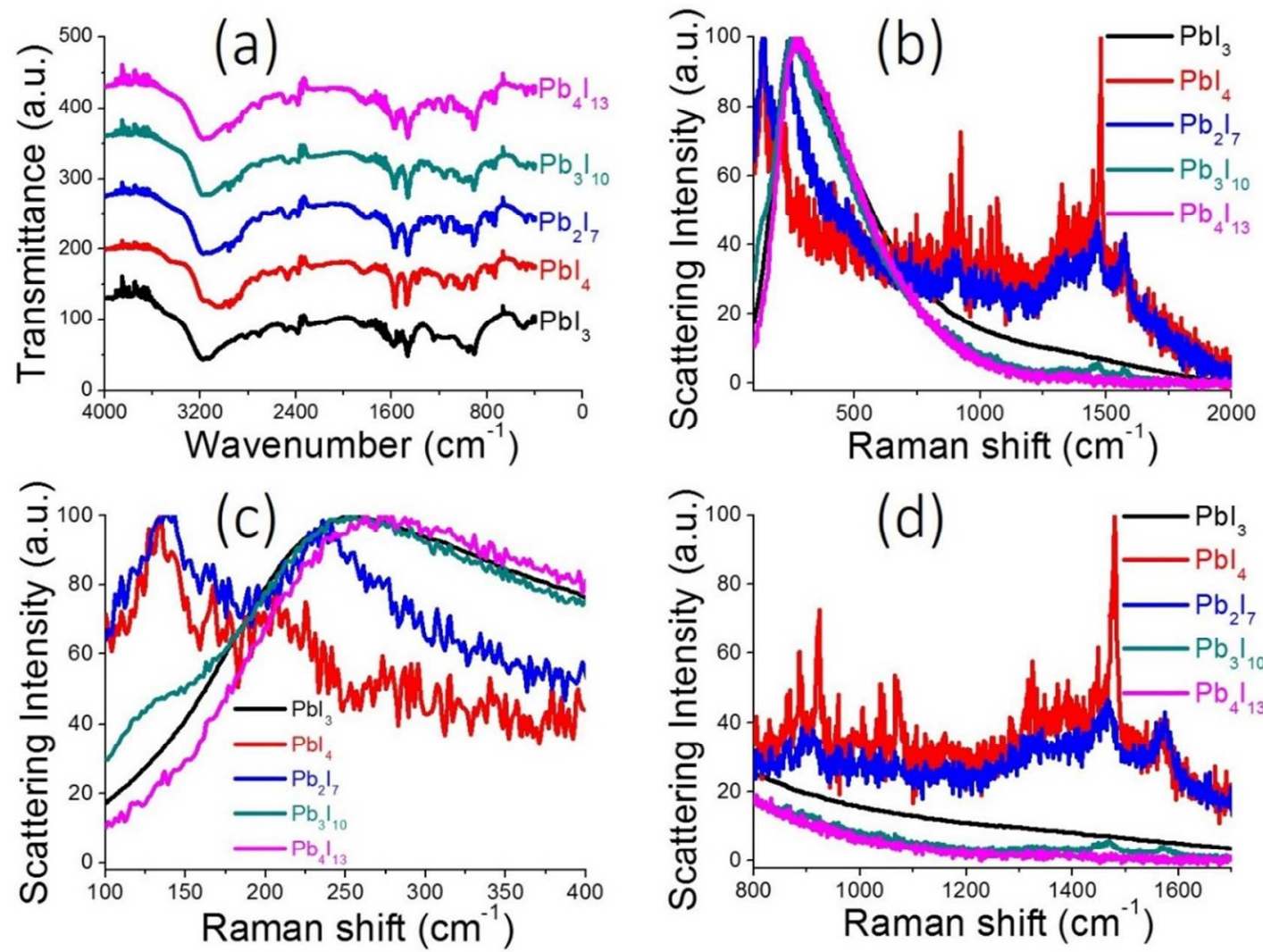

Figure S2. Vibrational spectra of (BA $)_{2}(\mathrm{MA})_{\mathrm{n}-1} \mathrm{~Pb}_{\mathrm{n}} \mathrm{I}_{3 \mathrm{n}+1}$ compounds: a) FT-IR and b) FT-Raman spectra, (c) and (d) represent close-up views of the low and high energy regions of the Raman spectra, respectively 


\section{S3. Complete crystallographic information}

\section{A. Non-centrosymmetric Refinements}

Table S1. Atomic coordinates $\left(\times 10^{4}\right)$ and equivalent isotropic displacement parameters $\left(\AA^{2} \times 10^{3}\right)$ for $(\mathrm{BA})_{2}(\mathrm{MA}) \mathrm{Pb}_{2} \mathrm{I}_{7}(\mathrm{n}=2)$ at $293(2) \mathrm{K}$ with estimated standard deviations in parentheses

\begin{tabular}{|c|c|c|c|c|c|}
\hline Label & $\mathrm{x}$ & $\mathrm{y}$ & z & Occupancy & $\mathrm{U}_{\mathrm{eq}}{ }^{*}$ \\
\hline $\mathrm{Pb}(1)$ & $7496(2)$ & $2489(5)$ & 2500 & 1 & $55(1)$ \\
\hline $\mathrm{Pb}(2)$ & $7492(3)$ & $4135(5)$ & 2500 & 1 & $68(1)$ \\
\hline $\mathrm{I}(1)$ & 10000 & $2441(5)$ & 0 & 1 & $122(2)$ \\
\hline $\mathrm{I}(2)$ & 5000 & $2600(5)$ & 0 & 1 & $101(2)$ \\
\hline $\mathrm{I}(3)$ & 5000 & $4031(5)$ & 0 & 1 & $132(2)$ \\
\hline $\mathrm{I}(4)$ & 10000 & $4222(5)$ & 0 & 1 & $108(2)$ \\
\hline $\mathrm{I}(5)$ & $7950(4)$ & $3308(5)$ & 2500 & 1 & $107(1)$ \\
\hline $\mathrm{I}(6)$ & $6902(8)$ & $4905(5)$ & 2500 & 1 & $147(3)$ \\
\hline $\mathrm{I}(7)$ & $6907(8)$ & $1718(5)$ & 2500 & 1 & $137(2)$ \\
\hline $\mathrm{N}(1)$ & $2350(100)$ & $3640(20)$ & 2500 & 1 & $220(30)$ \\
\hline $\mathrm{H}(1 \mathrm{~A})$ & 1676 & 3684 & 3213 & 0.5 & 329 \\
\hline $\mathrm{H}(1 \mathrm{~B})$ & 1984 & 3700 & 1603 & 0.5 & 329 \\
\hline $\mathrm{H}(1 \mathrm{C})$ & 3176 & 3761 & 2684 & 0.5 & 329 \\
\hline $\mathrm{C}(1)$ & $2710(100)$ & $3280(30)$ & 2500 & 1 & $220(30)$ \\
\hline $\mathrm{H}(1 \mathrm{D})$ & 3440 & 3231 & 1732 & 0.5 & 329 \\
\hline $\mathrm{H}(1 \mathrm{E})$ & 1821 & 3148 & 2301 & 0.5 & 329 \\
\hline $\mathrm{H}(1 \mathrm{~F})$ & 3107 & 3214 & 3467 & 0.5 & 329 \\
\hline $\mathrm{N}(2)$ & 2973 & 4669 & 2500 & 1 & $94(10)$ \\
\hline $\mathrm{H}(2 \mathrm{~A})$ & 2511 & 4469 & 2500 & 1 & 141 \\
\hline $\mathrm{H}(2 \mathrm{~B})$ & 3542 & 4687 & 3320 & 0.5 & 141 \\
\hline $\mathrm{H}(2 \mathrm{C})$ & 3542 & 4687 & 1680 & 0.5 & 141 \\
\hline$C(2)$ & 1851 & 4944 & 2500 & 1 & $100(300)$ \\
\hline $\mathrm{H}(2 \mathrm{D})$ & 1223 & 4931 & 3391 & 0.5 & 113 \\
\hline $\mathrm{H}(2 \mathrm{E})$ & 1223 & 4931 & 1609 & 0.5 & 113 \\
\hline
\end{tabular}




\begin{tabular}{|c|c|c|c|c|c|}
\hline$C(3)$ & 2791 & 5277 & 2500 & 1 & $100(300)$ \\
\hline $\mathrm{H}(3 \mathrm{~A})$ & 3433 & 5276 & 3383 & 0.5 & 113 \\
\hline $\mathrm{H}(3 \mathrm{~B})$ & 3433 & 5276 & 1617 & 0.5 & 113 \\
\hline$C(4)$ & 1881 & 5614 & 2500 & 1 & $100(300)$ \\
\hline $\mathrm{H}(4 \mathrm{~A})$ & 1254 & 5629 & 1610 & 0.5 & 113 \\
\hline $\mathrm{H}(4 \mathrm{~B})$ & 1254 & 5629 & 3390 & 0.5 & 113 \\
\hline$C(5)$ & 3041 & 5893 & 2500 & 1 & $100(300)$ \\
\hline $\mathrm{H}(5 \mathrm{~A})$ & 2552 & 6110 & 2500 & 1 & 141 \\
\hline $\mathrm{H}(5 \mathrm{~B})$ & 3654 & 5873 & 1615 & 0.5 & 141 \\
\hline $\mathrm{H}(5 \mathrm{C})$ & 3654 & 5873 & 3385 & 0.5 & 141 \\
\hline $\mathrm{N}(3)$ & 2884 & 1893 & 2500 & 1 & 615 \\
\hline $\mathrm{H}(3 \mathrm{C})$ & 2554 & 2107 & 2500 & 1 & 922 \\
\hline $\mathrm{H}(3 \mathrm{D})$ & 3436 & 1858 & 1680 & 0.5 & 922 \\
\hline $\mathrm{H}(3 \mathrm{E})$ & 3436 & 1858 & 3320 & 0.5 & 922 \\
\hline$C(6)$ & 1567 & 1652 & 2500 & 1 & 615 \\
\hline $\mathrm{H}(6 \mathrm{~A})$ & 954 & 1685 & 1609 & 0.5 & 738 \\
\hline $\mathrm{H}(6 \mathrm{~B})$ & 954 & 1685 & 3391 & 0.5 & 738 \\
\hline$C(7)$ & 2292 & 1298 & 2500 & 1 & 615 \\
\hline $\mathrm{H}(7 \mathrm{~A})$ & 2926 & 1278 & 3384 & 0.5 & 738 \\
\hline $\mathrm{H}(7 \mathrm{~B})$ & 2926 & 1278 & 1616 & 0.5 & 738 \\
\hline$C(8)$ & 1134 & 992 & 2500 & 1 & 615 \\
\hline $\mathrm{H}(8 \mathrm{~A})$ & 502 & 1005 & 3388 & 0.5 & 738 \\
\hline $\mathrm{H}(8 \mathrm{~B})$ & 502 & 1005 & 1612 & 0.5 & 738 \\
\hline$C(9)$ & 2002 & 654 & 2500 & 1 & 615 \\
\hline $\mathrm{H}(9 \mathrm{~A})$ & 1310 & 468 & 2500 & 1 & 922 \\
\hline $\mathrm{H}(9 \mathrm{~B})$ & 2620 & 642 & 3385 & 0.5 & 922 \\
\hline $\mathrm{H}(9 \mathrm{C})$ & 2620 & 642 & 1615 & 0.5 & 922 \\
\hline
\end{tabular}


Table S2. Anisotropic displacement parameters $\left(\AA^{2} \times 10^{3}\right)$ for $(\mathrm{BA})_{2}(\mathrm{MA}) \mathrm{Pb}_{2} \mathrm{I}_{7}(\mathrm{n}=2)$ at $293(2)$

$\mathrm{K}$ with estimated standard deviations in parentheses.

\begin{tabular}{|c|c|c|c|c|c|c|}
\hline Label & $\mathrm{U}_{11}$ & $\mathrm{U}_{22}$ & $\mathrm{U}_{33}$ & $\mathrm{U}_{12}$ & $\mathrm{U}_{13}$ & $\mathrm{U}_{23}$ \\
\hline $\mathrm{Pb}(1)$ & $42(1)$ & $78(1)$ & $46(1)$ & $6(1)$ & 0 & 0 \\
\hline $\mathrm{Pb}(2)$ & $50(2)$ & $95(2)$ & $59(1)$ & $10(1)$ & 0 & 0 \\
\hline $\mathrm{I}(1)$ & 101(3) & $157(5)$ & $108(2)$ & 0 & $65(3)$ & 0 \\
\hline $\mathrm{I}(2)$ & $78(3)$ & $146(5)$ & $78(2)$ & 0 & $-37(2)$ & 0 \\
\hline $\mathrm{I}(3)$ & $98(4)$ & $203(7)$ & $94(3)$ & 0 & $-57(3)$ & 0 \\
\hline $\mathrm{I}(4)$ & $71(3)$ & $179(6)$ & $75(2)$ & 0 & $30(2)$ & 0 \\
\hline $\mathrm{I}(5)$ & $99(2)$ & $74(2)$ & $149(3)$ & $14(4)$ & 0 & 0 \\
\hline $\mathrm{I}(6)$ & $97(4)$ & $70(3)$ & $274(9)$ & $-3(3)$ & 0 & 0 \\
\hline $\mathrm{I}(7)$ & $92(4)$ & $84(3)$ & $234(8)$ & $-6(4)$ & 0 & 0 \\
\hline
\end{tabular}

The anisotropic displacement factor exponent takes the form: $-2 \pi^{2}\left[h^{2} a^{* 2} U_{11}+\ldots+2 h_{k a}{ }^{*}{ }^{*} U_{12}\right]$.

Table S3. Selected bond lengths $[\AA]$ for $(B A)_{2}(\mathrm{MA}) \mathrm{Pb}_{2} \mathrm{I}_{7}(\mathrm{n}=2)$ at $293(2) \mathrm{K}$ with estimated standard deviations in parentheses.

\begin{tabular}{ll}
\hline Label & Distances \\
\hline $\mathrm{Pb}(1)-\mathrm{I}(7)$ & $3.081(7)$ \\
$\mathrm{Pb}(1)-\mathrm{I}(1)$ & $3.1561(14)$ \\
$\mathrm{Pb}(1)-\mathrm{I}(2)$ & $3.1749(16)$ \\
$\mathrm{Pb}(1)-\mathrm{I}(5)$ & $3.249(8)$ \\
$\mathrm{Pb}(2)-\mathrm{I}(6)$ & $3.075(6)$ \\
$\mathrm{Pb}(2)-\mathrm{I}(3)$ & $3.169(2)$ \\
$\mathrm{Pb}(2)-\mathrm{I}(4)$ & $3.1715(19)$ \\
$\mathrm{Pb}(2)-\mathrm{I}(5)$ & $3.278(8)$ \\
\hline
\end{tabular}


Table S4. Selected bond angles $\left[^{\circ}\right]$ for $(B A)_{2}(M A) \mathrm{Pb}_{2} \mathrm{I}_{7}(\mathrm{n}=2)$ at $293(2) \mathrm{K}$ with estimated standard deviations in parentheses.

\begin{tabular}{ll}
\hline Label & Angles \\
\hline $\mathrm{Pb}(1)^{\prime}-\mathrm{I}(1)-\mathrm{Pb}(1)$ & $173.1(3)$ \\
$\mathrm{Pb}(1)-\mathrm{I}(2)-\mathrm{Pb}(1)^{\prime}$ & $164.3(3)$ \\
$\mathrm{Pb}(2)-\mathrm{I}(3)-\mathrm{Pb}(2)^{\prime}$ & $165.1(4)$ \\
$\mathrm{Pb}(2)^{\prime}-\mathrm{I}(4)-\mathrm{Pb}(2)$ & $167.6(3)$ \\
$\mathrm{Pb}(1)-\mathrm{I}(5)-\mathrm{Pb}(2)$ & $165.62(11)$ \\
\hline
\end{tabular}


Table S5. Atomic coordinates $\left(\times 10^{4}\right)$ and equivalent isotropic displacement parameters $\left(\AA^{2} \times 10^{3}\right)$ for $(\mathrm{BA})_{2}(\mathrm{MA})_{2} \mathrm{~Pb}_{3} \mathrm{I}_{10}(\mathrm{n}=3)$ at $293(2) \mathrm{K}$ with estimated standard deviations in parentheses.

\begin{tabular}{|c|c|c|c|c|c|}
\hline Label & $\mathrm{x}$ & $\mathrm{y}$ & $\mathrm{z}$ & Occupancy & $\mathrm{U}_{\mathrm{eq}}{ }^{*}$ \\
\hline $\mathrm{Pb}(1)$ & $5147(4)$ & 0 & 5000 & 1 & $48(1)$ \\
\hline $\mathrm{Pb}(2)$ & $5246(2)$ & $1239(1)$ & $5010(2)$ & 1 & $53(1)$ \\
\hline $\mathrm{I}(1)$ & $5192(15)$ & $605(1)$ & $4673(4)$ & 1 & $103(2)$ \\
\hline $\mathrm{I}(2)$ & $7790(7)$ & $1278(1)$ & $2559(8)$ & 1 & $102(2)$ \\
\hline I(3) & $7770(8)$ & $1158(1)$ & $7460(8)$ & 1 & $100(2)$ \\
\hline I(4) & $2783(17)$ & $-43(1)$ & $2510(20)$ & 1 & $189(3)$ \\
\hline $\mathrm{I}(5)$ & $5265(19)$ & $1817(1)$ & $5588(4)$ & 1 & $138(2)$ \\
\hline $\mathrm{N}(1)$ & $10900(120)$ & $628(18)$ & $5390(100)$ & 1 & $230(40)$ \\
\hline $\mathrm{H}(1 \mathrm{~A})$ & 10863 & 783 & 5818 & 1 & 350 \\
\hline $\mathrm{H}(1 \mathrm{~B})$ & 11724 & 615 & 4835 & 1 & 350 \\
\hline $\mathrm{H}(1 \mathrm{C})$ & 10903 & 507 & 6101 & 1 & 350 \\
\hline $\mathrm{C}(1)$ & $9610(130)$ & $590(20)$ & $4450(130)$ & 1 & $230(40)$ \\
\hline $\mathrm{H}(1 \mathrm{D})$ & 8721 & 605 & 5051 & 1 & 350 \\
\hline $\mathrm{H}(1 \mathrm{E})$ & 9660 & 429 & 3970 & 1 & 350 \\
\hline $\mathrm{H}(1 \mathrm{~F})$ & 9594 & 727 & 3695 & 1 & 350 \\
\hline $\mathrm{N}(2)$ & 4984 & 1644 & 9592 & 1 & $130(16)$ \\
\hline $\mathrm{H}(2 \mathrm{~A})$ & 5693 & 1549 & 9158 & 1 & 195 \\
\hline $\mathrm{H}(2 \mathrm{~B})$ & 4282 & 1682 & 8917 & 1 & 195 \\
\hline $\mathrm{H}(2 \mathrm{C})$ & 4574 & 1555 & 10346 & 1 & 195 \\
\hline$C(2)$ & 5705 & 1903 & 10231 & 1 & $130(16)$ \\
\hline $\mathrm{H}(2 \mathrm{D})$ & 6481 & 1860 & 10955 & 1 & 156 \\
\hline $\mathrm{H}(2 \mathrm{E})$ & 6166 & 1997 & 9411 & 1 & 156 \\
\hline$C(3)$ & 4519 & 2072 & 10990 & 1 & $130(16)$ \\
\hline $\mathrm{H}(3 \mathrm{~A})$ & 3661 & 2092 & 10331 & 1 & 156 \\
\hline $\mathrm{H}(3 \mathrm{~B})$ & 4184 & 1992 & 11919 & 1 & 156 \\
\hline $\mathrm{C}(4)$ & 5201 & 2325 & 11309 & 1 & $130(16)$ \\
\hline $\mathrm{H}(4 \mathrm{~A})$ & 5255 & 2427 & 10396 & 1 & 156 \\
\hline $\mathrm{H}(4 \mathrm{~B})$ & 6206 & 2304 & 11705 & 1 & 156 \\
\hline$C(5)$ & 4187 & 2458 & 12485 & 1 & $130(16)$ \\
\hline $\mathrm{H}(5 \mathrm{~A})$ & 4590 & 2625 & 12726 & 1 & 195 \\
\hline
\end{tabular}




\begin{tabular}{llllll}
$\mathrm{H}(5 \mathrm{~B})$ & 4143 & 2355 & 13381 & 1 & 195 \\
$\mathrm{H}(5 \mathrm{C})$ & 3197 & 2478 & 12079 & 1 & 195 \\
\hline
\end{tabular}

" $\mathrm{U}_{\mathrm{eq}}$ is defined as one third of the trace of the orthogonalized $\mathrm{U}_{\mathrm{ij}}$ tensor.

Table S6. Anisotropic displacement parameters $\left(\AA^{2} \times 10^{3}\right)$ for $(\mathrm{BA})_{2}(\mathrm{MA})_{2} \mathrm{~Pb}_{3} \mathrm{I}_{10}(\mathrm{n}=3)$ at 293(2) K with estimated standard deviations in parentheses.

\begin{tabular}{lllllll}
\hline Label & $\mathrm{U}_{11}$ & $\mathrm{U}_{22}$ & $\mathrm{U}_{33}$ & $\mathrm{U}_{12}$ & $\mathrm{U}_{13}$ & $\mathrm{U}_{23}$ \\
\hline $\mathrm{Pb}(1)$ & $41(2)$ & $63(1)$ & $41(1)$ & 0 & 0 & $-1(1)$ \\
$\mathrm{Pb}(2)$ & $52(1)$ & $66(1)$ & $41(1)$ & $3(2)$ & $16(1)$ & $-1(1)$ \\
$\mathrm{I}(1)$ & $144(3)$ & $53(2)$ & $112(2)$ & $-4(4)$ & $-25(5)$ & $0(2)$ \\
$\mathrm{I}(2)$ & $77(3)$ & $161(3)$ & $68(2)$ & $-20(5)$ & $38(2)$ & $-22(4)$ \\
$\mathrm{I}(3)$ & $73(3)$ & $154(3)$ & $72(2)$ & $19(5)$ & $-39(2)$ & $-4(5)$ \\
$\mathrm{I}(4)$ & $219(6)$ & $129(3)$ & $219(5)$ & $16(11)$ & $-184(5)$ & $-5(11)$ \\
$\mathrm{I}(5)$ & $264(6)$ & $58(2)$ & $93(2)$ & $-10(8)$ & $22(6)$ & $-2(2)$ \\
\hline
\end{tabular}

The anisotropic displacement factor exponent takes the form: $-2 \pi^{2}\left[h^{2} a^{2}{ }^{2} U_{11}+\ldots+2 h k a b{ }^{*} U_{12}\right]$.

Table S7. Selected bond lengths $[\AA]$ for $(\mathrm{BA})_{2}(\mathrm{MA})_{2} \mathrm{~Pb}_{3} \mathrm{I}_{10}(\mathrm{n}=3)$ at $293(2) \mathrm{K}$ with estimated standard deviations in parentheses.

\begin{tabular}{ll}
\hline Label & Distances \\
\hline $\mathrm{Pb}(1)-\mathrm{I}(4)$ & $3.065(8)$ \\
$\mathrm{Pb}(1)-\mathrm{I}(1)$ & $3.157(3)$ \\
$\mathrm{Pb}(1)-\mathrm{I}(4)$ & $3.249(8)$ \\
$\mathrm{Pb}(2)-\mathrm{I}(5)$ & $3.047(4)$ \\
$\mathrm{Pb}(2)-\mathrm{I}(2)$ & $3.152(6)$ \\
$\mathrm{Pb}(2)-\mathrm{I}(3)$ & $3.160(6)$ \\
$\mathrm{Pb}(2)-\mathrm{I}(2)$ & $3.170(6)$ \\
$\mathrm{Pb}(2)-\mathrm{I}(3)$ & $3.179(6)$ \\
$\mathrm{Pb}(2)-\mathrm{I}(1)$ & $3.309(3)$ \\
\hline
\end{tabular}


Table S8. Selected bond angles $\left[{ }^{\circ}\right]$ for $(\mathrm{BA})_{2}(\mathrm{MA})_{2} \mathrm{~Pb}_{3} \mathrm{I}_{10}(\mathrm{n}=3)$ at $293(2) \mathrm{K}$ with estimated standard deviations in parentheses.

\begin{tabular}{ll}
\hline Label & Angles \\
\hline $\mathrm{Pb}(1)-\mathrm{I}(1)-\mathrm{Pb}(2)$ & $169.52(12)$ \\
$\mathrm{Pb}(2)-\mathrm{I}(2)-\mathrm{Pb}(2)^{\prime}$ & $172.28(17)$ \\
$\mathrm{Pb}(2)-\mathrm{I}(3)-\mathrm{Pb}(2)^{\prime}$ & $164.57(15)$ \\
$\mathrm{Pb}(1)-\mathrm{I}(4)-\mathrm{Pb}(1)^{\prime}$ & $171.3(3)$ \\
\hline
\end{tabular}

Table S9. Atomic coordinates $\left(\mathrm{x} 10^{4}\right)$ and equivalent isotropic displacement parameters $\left(\AA^{2} \times 10^{3}\right)$ for $(\mathrm{BA})_{2}(\mathrm{MA})_{3} \mathrm{~Pb}_{4} \mathrm{I}_{13}(\mathrm{n}=4)$ at $293(2) \mathrm{K}$ with estimated standard deviations in parentheses

\begin{tabular}{llllll}
\hline Label & $\mathrm{x}$ & $\mathrm{y}$ & $\mathrm{z}$ & Occupancy & $\mathrm{U}_{\mathrm{eq}}{ }^{*}$ \\
\hline $\mathrm{Pb}(1)$ & $2502(3)$ & $1997(3)$ & 2500 & 1 & $42(1)$ \\
$\mathrm{Pb}(2)$ & $2502(2)$ & $2983(3)$ & 2500 & 1 & $41(1)$ \\
$\mathrm{Pb}(3)$ & $2497(2)$ & $1007(3)$ & 2500 & 1 & $48(1)$ \\
$\mathrm{Pb}(4)$ & $2485(2)$ & $3976(3)$ & 2500 & 1 & $45(1)$ \\
$\mathrm{I}(1)$ & 5000 & $2923(3)$ & 5000 & 1 & $101(2)$ \\
$\mathrm{I}(2)$ & 0 & $1949(3)$ & 5000 & 1 & $105(3)$ \\
$\mathrm{I}(3)$ & $2886(9)$ & $1513(3)$ & 2500 & 1 & $114(3)$ \\
$\mathrm{I}(4)$ & $2104(4)$ & $2479(3)$ & 2500 & 1 & $104(2)$ \\
$\mathrm{I}(5)$ & $2965(7)$ & $3463(3)$ & 2500 & 1 & $95(2)$ \\
$\mathrm{I}(6)$ & 5000 & $2030(3)$ & 5000 & 1 & $124(3)$ \\
$\mathrm{I}(7)$ & 0 & $3902(3)$ & 5000 & 1 & $100(2)$ \\
$\mathrm{I}(8)$ & 5000 & $966(3)$ & 5000 & 1 & $100(2)$ \\
$\mathrm{I}(9)$ & 5000 & $4007(3)$ & 5000 & 1 & $90(2)$ \\
$\mathrm{I}(10)$ & $1905(8)$ & $538(3)$ & 2500 & 1 & $121(3)$ \\
$\mathrm{I}(11)$ & $1898(10)$ & $4437(3)$ & 2500 & 1 & $139(4)$ \\
$\mathrm{I}(12)$ & 0 & $1069(3)$ & 5000 & 1 & $88(2)$ \\
$\mathrm{I}(13)$ & 0 & $3014(3)$ & 5000 & 1 & $119(3)$ \\
$\mathrm{N}(1)$ & $3260(90)$ & $1476(14)$ & 7500 & 1 & $200(30)$ \\
$\mathrm{H}(1 \mathrm{~A})$ & 3694 & 1527 & 6678 & 0.5 & 301 \\
$\mathrm{H}(1 \mathrm{~B})$ & 3364 & 1338 & 7508 & 0.5 & 301 \\
$\mathrm{H}(1 \mathrm{C})$ & 3698 & 1529 & 8314 & 0.5 & 301
\end{tabular}




\begin{tabular}{|c|c|c|c|c|c|}
\hline$C(1)$ & 1410(110) & $1538(16)$ & 7500 & 1 & $200(30)$ \\
\hline $\mathrm{H}(1 \mathrm{D})$ & 1168 & 1611 & 8411 & 0.5 & 301 \\
\hline $\mathrm{H}(1 \mathrm{E})$ & 823 & 1413 & 7440 & 0.5 & 301 \\
\hline $\mathrm{H}(1 \mathrm{~F})$ & 1193 & 1625 & 6649 & 0.5 & 301 \\
\hline $\mathrm{N}(2)$ & $1990(60)$ & $2569(10)$ & 7500 & 1 & $160(20)$ \\
\hline $\mathrm{H}(2 \mathrm{~A})$ & 1714 & 2594 & 8445 & 0.5 & 236 \\
\hline $\mathrm{H}(2 \mathrm{~B})$ & 1267 & 2500 & 7026 & 0.5 & 236 \\
\hline $\mathrm{H}(2 \mathrm{C})$ & 2162 & 2688 & 7029 & 0.5 & 236 \\
\hline$C(2)$ & $3300(60)$ & $2449(15)$ & 7500 & 1 & $160(20)$ \\
\hline $\mathrm{H}(2 \mathrm{D})$ & 4156 & 2540 & 7499 & 1 & 236 \\
\hline $\mathrm{H}(2 \mathrm{E})$ & 3320 & 2363 & 6618 & 0.5 & 236 \\
\hline $\mathrm{H}(2 \mathrm{~F})$ & 3321 & 2363 & 8383 & 0.5 & 236 \\
\hline $\mathrm{N}(3)$ & $2170(60)$ & $3529(8)$ & 7500 & 1 & $102(13)$ \\
\hline $\mathrm{H}(3 \mathrm{~A})$ & 2445 & 3607 & 8283 & 0.5 & 153 \\
\hline $\mathrm{H}(3 \mathrm{~B})$ & 1196 & 3501 & 7566 & 0.5 & 153 \\
\hline $\mathrm{H}(3 \mathrm{C})$ & 2348 & 3598 & 6651 & 0.5 & 153 \\
\hline$C(3)$ & $3000(70)$ & $3341(9)$ & 7500 & 1 & $102(13)$ \\
\hline $\mathrm{H}(3 \mathrm{D})$ & 3554 & 3329 & 8423 & 0.5 & 153 \\
\hline $\mathrm{H}(3 \mathrm{E})$ & 3684 & 3340 & 6665 & 0.5 & 153 \\
\hline $\mathrm{H}(3 \mathrm{~F})$ & 2326 & 3225 & 7411 & 0.5 & 153 \\
\hline $\mathrm{N}(4)$ & 2486 & 4279 & 7500 & 1 & $119(17)$ \\
\hline $\mathrm{H}(4 \mathrm{~A})$ & 1639 & 4207 & 7500 & 1 & 178 \\
\hline $\mathrm{H}(4 \mathrm{~B})$ & 3016 & 4248 & 8318 & 0.5 & 178 \\
\hline $\mathrm{H}(4 \mathrm{C})$ & 3016 & 4248 & 6682 & 0.5 & 178 \\
\hline $\mathrm{C}(4)$ & 2129 & 4507 & 7500 & 1 & $100(1200)$ \\
\hline $\mathrm{H}(4 \mathrm{D})$ & 1526 & 4538 & 6619 & 0.5 & 143 \\
\hline $\mathrm{H}(4 \mathrm{E})$ & 1526 & 4538 & 8381 & 0.5 & 143 \\
\hline $\mathrm{C}(5)$ & 3428 & 4645 & 7500 & 1 & $100(1200)$ \\
\hline $\mathrm{H}(5 \mathrm{~A})$ & 4039 & 4623 & 6611 & 0.5 & 143 \\
\hline $\mathrm{H}(5 \mathrm{~B})$ & 4039 & 4623 & 8389 & 0.5 & 143 \\
\hline$C(6)$ & 2730 & 4870 & 7500 & 1 & $100(1200)$ \\
\hline $\mathrm{H}(6 \mathrm{~A})$ & 2100 & 4886 & 6618 & 0.5 & 143 \\
\hline $\mathrm{H}(6 \mathrm{~B})$ & 2100 & 4886 & 8382 & 0.5 & 143 \\
\hline$C(7)$ & 3901 & 5039 & 7500 & 1 & $100(1200)$ \\
\hline $\mathrm{H}(7 \mathrm{~A})$ & 3420 & 5172 & 7500 & 1 & 178 \\
\hline $\mathrm{H}(7 \mathrm{~B})$ & 4515 & 5026 & 6617 & 0.5 & 178 \\
\hline
\end{tabular}




\begin{tabular}{|c|c|c|c|c|c|}
\hline $\mathrm{H}(7 \mathrm{C})$ & 4515 & 5026 & 8383 & 0.5 & 178 \\
\hline $\mathrm{N}(5)$ & 2558 & 777 & 7500 & 1 & $900(300)$ \\
\hline $\mathrm{H}(5 \mathrm{C})$ & 1701 & 847 & 7500 & 1 & 1361 \\
\hline $\mathrm{H}(5 \mathrm{D})$ & 3084 & 809 & 6682 & 0.5 & 1361 \\
\hline $\mathrm{H}(5 \mathrm{E})$ & 3084 & 809 & 8318 & 0.5 & 1361 \\
\hline$C(8)$ & 2229 & 548 & 7500 & 1 & $900(1200)$ \\
\hline $\mathrm{H}(8 \mathrm{~A})$ & 1612 & 520 & 8376 & 0.5 & 1089 \\
\hline $\mathrm{H}(8 \mathrm{~B})$ & 1612 & 520 & 6624 & 0.5 & 1089 \\
\hline$C(9)$ & 3449 & 391 & 7500 & 1 & $900(1200)$ \\
\hline $\mathrm{H}(9 \mathrm{~A})$ & 4073 & 407 & 8388 & 0.5 & 1089 \\
\hline $\mathrm{H}(9 \mathrm{~B})$ & 4073 & 407 & 6612 & 0.5 & 1089 \\
\hline$C(10)$ & 2691 & 183 & 7500 & 1 & $900(1200)$ \\
\hline $\mathrm{H}(10 \mathrm{~A})$ & 2051 & 175 & 6619 & 0.5 & 1089 \\
\hline $\mathrm{H}(10 \mathrm{~B})$ & 2051 & 175 & 8381 & 0.5 & 1089 \\
\hline$C(11)$ & 3753 & -7 & 7500 & 1 & $900(1200)$ \\
\hline $\mathrm{H}(11 \mathrm{~A})$ & 3170 & -132 & 7500 & 1 & 1361 \\
\hline $\mathrm{H}(11 \mathrm{~B})$ & 4373 & -3 & 8383 & 0.5 & 1361 \\
\hline $\mathrm{H}(11 \mathrm{C})$ & 4373 & -3 & 6617 & 0.5 & 1361 \\
\hline
\end{tabular}

\footnotetext{
${ }^{*} U_{\text {eq }}$ is defined as one third of the trace of the orthogonalized $U_{i j}$ tensor.
} 
Table S10. Anisotropic displacement parameters $\left(\AA^{2} \times 10^{3}\right)$ for $(\mathrm{BA})_{2}(\mathrm{MA})_{3} \mathrm{~Pb}_{4} \mathrm{I}_{13}(\mathrm{n}=4)$ at 293(2) K with estimated standard deviations in parentheses.

\begin{tabular}{lllllll}
\hline Label & $\mathrm{U}_{11}$ & $\mathrm{U}_{22}$ & $\mathrm{U}_{33}$ & $\mathrm{U}_{12}$ & $\mathrm{U}_{13}$ & $\mathrm{U}_{23}$ \\
\hline $\mathrm{Pb}(1)$ & $36(2)$ & $39(2)$ & $51(2)$ & $4(1)$ & 0 & 0 \\
$\mathrm{~Pb}(2)$ & $36(2)$ & $55(2)$ & $34(2)$ & $3(1)$ & 0 & 0 \\
$\mathrm{~Pb}(3)$ & $44(2)$ & $52(2)$ & $49(2)$ & $5(2)$ & 0 & 0 \\
$\mathrm{~Pb}(4)$ & $33(2)$ & $56(2)$ & $46(2)$ & $8(1)$ & 0 & 0 \\
$\mathrm{I}(1)$ & $76(3)$ & $152(6)$ & $74(4)$ & 0 & $-31(3)$ & 0 \\
$\mathrm{I}(2)$ & $75(3)$ & $168(7)$ & $71(4)$ & 0 & $48(3)$ & 0 \\
$\mathrm{I}(3)$ & $115(4)$ & $43(3)$ & $184(9)$ & $3(4)$ & 0 & 0 \\
$\mathrm{I}(4)$ & $106(2)$ & $30(2)$ & $176(4)$ & $7(4)$ & 0 & 0 \\
$\mathrm{I}(5)$ & $86(3)$ & $32(3)$ & $169(7)$ & $2(3)$ & 0 & 0 \\
$\mathrm{I}(6)$ & $117(4)$ & $116(5)$ & $140(6)$ & 0 & $-108(4)$ & 0 \\
$\mathrm{I}(7)$ & $61(3)$ & $164(7)$ & $75(4)$ & 0 & $28(3)$ & 0 \\
$\mathrm{I}(8)$ & $78(4)$ & $146(7)$ & $74(4)$ & 0 & $-35(3)$ & 0 \\
$\mathrm{I}(9)$ & $54(3)$ & $149(6)$ & $67(4)$ & 0 & $-32(3)$ & 0 \\
$\mathrm{I}(10)$ & $69(3)$ & $48(3)$ & $245(10)$ & $0(3)$ & 0 & 0 \\
$\mathrm{I}(11)$ & $96(4)$ & $55(3)$ & $265(13)$ & $8(4)$ & 0 & 0 \\
$\mathrm{I}(12)$ & $73(3)$ & $127(6)$ & $62(3)$ & 0 & $39(3)$ & 0 \\
$\mathrm{I}(13)$ & $112(5)$ & $128(6)$ & $117(6)$ & 0 & $78(4)$ & 0 \\
\hline $\mathrm{Th}$
\end{tabular}

The anisotropic displacement factor exponent takes the form: $-2 \pi^{2}\left[h^{2} a^{* 2} U_{11}+\ldots+2 h k a b^{*} U_{12}\right]$. 
Table S11. Selected bond lengths $[\AA]$ for $(B A)_{2}(M A)_{3} \mathrm{~Pb}_{4} \mathrm{I}_{13}(\mathrm{n}=4)$ at $293(2) \mathrm{K}$ with estimated standard deviations in parentheses.

\begin{tabular}{ll}
\hline Label & Distances \\
\hline $\mathrm{Pb}(1)-\mathrm{I}(4)$ & $3.12(2)$ \\
$\mathrm{Pb}(1)-\mathrm{I}(3)$ & $3.14(2)$ \\
$\mathrm{Pb}(1)-\mathrm{I}(6)$ & $3.154(8)$ \\
$\mathrm{Pb}(1)-\mathrm{I}(2)$ & $3.164(8)$ \\
$\mathrm{Pb}(2)-\mathrm{I}(5)$ & $3.122(17)$ \\
$\mathrm{Pb}(2)-\mathrm{I}(13)$ & $3.156(8)$ \\
$\mathrm{Pb}(2)-\mathrm{I}(1)$ & $3.170(8)$ \\
$\mathrm{Pb}(2)-\mathrm{I}(4)$ & $3.26(2)$ \\
$\mathrm{Pb}(3)-\mathrm{I}(10)$ & $3.06(2)$ \\
$\mathrm{Pb}(3)-\mathrm{I}(8)$ & $3.161(8)$ \\
$\mathrm{Pb}(3)-\mathrm{I}(12)$ & $3.172(9)$ \\
$\mathrm{Pb}(3)-\mathrm{I}(3)$ & $3.27(2)$ \\
$\mathrm{Pb}(4)-\mathrm{I}(11)$ & $3.01(2)$ \\
$\mathrm{Pb}(4)-\mathrm{I}(9)$ & $3.164(8)$ \\
$\mathrm{Pb}(4)-\mathrm{I}(7)$ & $3.175(9)$ \\
$\mathrm{Pb}(4)-\mathrm{I}(5)$ & $3.330(15)$ \\
\hline
\end{tabular}


Table S12. Selected bond angles $\left[^{\circ}\right]$ for $(\mathrm{BA})_{2}(\mathrm{MA})_{3} \mathrm{~Pb}_{4} \mathrm{I}_{13}(\mathrm{n}=4)$ at $293(2) \mathrm{K}$ with estimated standard deviations in parentheses.

\begin{tabular}{ll}
\hline Label & Angles \\
\hline $\mathrm{Pb}(2)^{\prime}-\mathrm{I}(1)-\mathrm{Pb}(2)$ & $166.1(12)$ \\
$\mathrm{Pb}(1)-\mathrm{I}(2)-\mathrm{Pb}(1)^{\prime}$ & $168.8(14)$ \\
$\mathrm{Pb}(1)-\mathrm{I}(3)-\mathrm{Pb}(3)$ & $167.6(12)$ \\
$\mathrm{Pb}(1)-\mathrm{I}(4)-\mathrm{Pb}(2)$ & $167.2(6)$ \\
$\mathrm{Pb}(2)-\mathrm{I}(5)-\mathrm{Pb}(4)$ & $165.0(9)$ \\
$\mathrm{Pb}(1)-\mathrm{I}(6)-\mathrm{Pb}(1)^{\prime}$ & $172.4(12)$ \\
$\mathrm{Pb}(4)-\mathrm{I}(7)-\mathrm{Pb}(4)^{\prime}$ & $162.6(14)$ \\
$\mathrm{Pb}(3)^{\prime}-\mathrm{I}(8)-\mathrm{Pb}(3)$ & $170.4(14)$ \\
$\mathrm{Pb}(4)^{\prime}-\mathrm{I}(9)-\mathrm{Pb}(4)$ & $172.9(14)$ \\
$\mathrm{Pb}(3)-\mathrm{I}(12)-\mathrm{Pb}(3)^{\prime}$ & $165.5(13)$ \\
$\mathrm{Pb}(2)-\mathrm{I}(13)-\mathrm{Pb}(2)^{\prime}$ & $172.7(14)$ \\
\hline
\end{tabular}




\section{B. Centrosymmetric Refinements}

Table S13. Crystal data and structure refinement for $(\mathrm{BA})_{2}(\mathrm{MA})_{\mathrm{n}-1} \mathrm{~Pb}_{\mathrm{n}} \mathrm{I}_{3 \mathrm{n}+1}(\mathrm{n}=2-4)$ at $293(2) \mathrm{K}$ in centrosymmetric space groups

\begin{tabular}{|c|c|c|c|}
\hline Empirical formula & $(\mathrm{BA})_{2}(\mathrm{MA}) \mathrm{Pb}_{2} \mathrm{I}_{7}(\mathrm{n}=2)$ & $(\mathrm{BA})_{2}(\mathrm{MA})_{2} \mathrm{~Pb}_{3} \mathrm{I}_{10}(\mathrm{n}=3)$ & $(\mathrm{BA})_{2}(\mathrm{MA})_{3} \mathrm{~Pb}_{4} \mathrm{I}_{13}(\mathrm{n}=4)$ \\
\hline Formula weight & 1483.04 & 2103.00 & 2722.95 \\
\hline Temperature & & $293(2) \mathrm{K}$ & \\
\hline Wavelength & & $0.71073 \AA$ & \\
\hline Refinement method & & Full-matrix least-squares on $\mathrm{F}^{2}$ & \\
\hline Crystal system & orthorhombic & orthorhombic & orthorhombic \\
\hline Space group & $\mathrm{Ccmm}$ & Acam & $\mathrm{Ccmm}$ \\
\hline 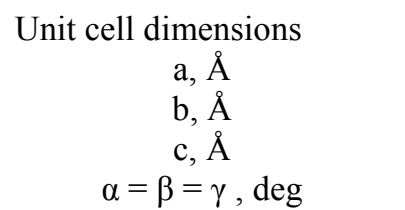 & $\begin{array}{l}8.9470(4) \\
39.347(2) \\
8.8589(6) \\
90^{\circ}\end{array}$ & $\begin{array}{l}8.9275(6) \\
51.959(4) \\
8.8777(6) \\
90^{\circ}\end{array}$ & $\begin{array}{l}8.9274(4) \\
64.383(3) \\
8.8816(4) \\
90^{\circ}\end{array}$ \\
\hline Volume, $\AA^{3}$ & $3118.7(3)$ & $4118.0(5)$ & $5104.9(4)$ \\
\hline Z & 4 & 4 & 4 \\
\hline Density (calc), $\mathrm{g} / \mathrm{cm}^{3}$ & 3.159 & 3.392 & 3.543 \\
\hline Absorption coeff., $\mathrm{mm}^{-1}$ & 17.712 & 19.739 & 21.026 \\
\hline $\mathrm{F}(000)$ & 2560 & 3600 & 4640 \\
\hline Crystal size $\left(\mathrm{mm}^{3}\right)$ & $0.140 \times 0.081 \times 0.025$ & $0.098 \times 0.092 \times 0.052$ & $0.079 \times 0.073 \times 0.009$ \\
\hline$\theta$ range & 2.521 to $25.000^{\circ}$ & 1.568 to $24.996^{\circ}$ & 2.471 to $24.999^{\circ}$ \\
\hline Completeness to $\theta$ & $99.7 \%$ & $99.9 \%$ & $99.4 \%$ \\
\hline Index ranges & $\begin{array}{c}-10<=\mathrm{h}<=10 \\
-46<=\mathrm{k}<=46 \\
-10<=1<=10\end{array}$ & $\begin{array}{c}-10<=\mathrm{h}<=10 \\
-61<=\mathrm{k}<=61, \\
-10<=1<=10\end{array}$ & $\begin{array}{c}-10<=\mathrm{h}<=10 \\
-76<=\mathrm{k}<=76 \\
-10<=1<=10\end{array}$ \\
\hline Reflections collected & 9452 & 12858 & 13717 \\
\hline Independent reflections & $1378\left[\mathrm{R}_{\mathrm{int}}=0.0365\right]$ & $1952\left[\mathrm{R}_{\mathrm{int}}=0.0722\right]$ & $2428\left[\mathrm{R}_{\mathrm{int}}=0.0506\right]$ \\
\hline $\begin{array}{l}\text { Data / restraints / } \\
\text { parameters }\end{array}$ & $1378 / 2 / 42$ & $1952 / 2 / 54$ & $2428 / 3 / 69$ \\
\hline Goodness-of-fit & 1.283 & 1.202 & 1.055 \\
\hline $\begin{array}{l}\text { Final } R \text { indices } \\
{[I>2 \sigma(I)]}\end{array}$ & $\begin{array}{l}\mathrm{R}_{\mathrm{obs}}=0.0663 \\
\mathrm{wR}_{\mathrm{obs}}=0.1592 \\
\mathrm{R}_{\mathrm{all}}=0.0706 \\
\mathrm{wR}_{\mathrm{all}}=0.1619\end{array}$ & $\begin{array}{l}\mathrm{R}_{\mathrm{obs}}=0.0982 \\
\mathrm{wR}_{\mathrm{obs}}=0.2132 \\
\mathrm{R}_{\mathrm{all}}=0.1272 \\
\mathrm{wR}_{\mathrm{all}}=0.2292\end{array}$ & $\begin{array}{l}\mathrm{R}_{\mathrm{obs}}=0.0496 \\
\mathrm{wR}_{\mathrm{obs}}=0.1312 \\
\mathrm{R}_{\mathrm{all}}=0.0854 \\
\mathrm{wR}_{\mathrm{all}}=0.1489\end{array}$ \\
\hline $\begin{array}{l}\text { Largest diff. peak and hole } \\
\left(\mathrm{e} \cdot \AA^{-3}\right)\end{array}$ & 1.303 and -2.310 & 3.593 and -2.589 & 1.217 and -2.350 \\
\hline \multicolumn{4}{|c|}{$\begin{array}{l}\mathrm{R}=\Sigma|| \mathrm{F}_{\mathrm{o}}|-| \mathrm{F}_{\mathrm{c}} \| / \Sigma\left|\mathrm{F}_{\mathrm{o}}\right|, \mathrm{wR}=\left\{\Sigma\left[\mathrm{w}\left(\left|\mathrm{F}_{\mathrm{o}}\right|^{2}-\left|\mathrm{F}_{\mathrm{c}}\right|^{2}\right)^{2}\right] / \Sigma\left[\mathrm{w}\left(\left|\mathrm{F}_{\mathrm{o}}\right|^{4}\right)\right]\right\}^{1 / 2} \text { and } \\
\mathrm{n}=2, \mathrm{w}=1 /\left[\sigma^{2}\left(\mathrm{Fo}^{2}\right)+(0.0588 \mathrm{P})^{2}+70.7427 \mathrm{P}\right] \\
\mathrm{n}=3, \mathrm{w}=1 /\left[\sigma^{2}\left(\mathrm{Fo}^{2}\right)+(0.0795 \mathrm{P})^{2}+255.6964 \mathrm{P}\right] \\
\mathrm{n}=4, \mathrm{w}=1 /\left[\sigma^{2}\left(\mathrm{Fo}^{2}\right)+(0.0669 \mathrm{P})^{2}+98.3113 \mathrm{P}\right], \text { where } \mathrm{P}=\left(\mathrm{Fo}^{2}+2 \mathrm{Fc}^{2}\right) / 3\end{array}$} \\
\hline
\end{tabular}


Table S14. Atomic coordinates $\left(\times 10^{4}\right)$ and equivalent isotropic displacement parameters $\left(\AA^{2} \times 10^{3}\right)$ for $(\mathrm{BA})_{2}(\mathrm{MA}) \mathrm{Pb}_{2} \mathrm{I}_{7}(\mathrm{n}=2)$ at $293(2) \mathrm{K}$ with estimated standard deviations in parentheses.

\begin{tabular}{|c|c|c|c|c|c|}
\hline Label & $\mathrm{x}$ & $\mathrm{y}$ & $\mathrm{z}$ & Occupancy & $\mathrm{U}_{\mathrm{eq}}{ }^{*}$ \\
\hline $\mathrm{Pb}(1)$ & $2507(1)$ & $823(1)$ & 7500 & 1 & $60(1)$ \\
\hline $\mathrm{I}(1)$ & 2051(4) & 0 & 7500 & 1 & $104(2)$ \\
\hline $\mathrm{I}(2)$ & 5000 & $714(1)$ & 5000 & 1 & $113(1)$ \\
\hline $\mathrm{I}(3)$ & 0 & $893(1)$ & 5000 & 1 & $112(1)$ \\
\hline I(4) & $3101(3)$ & $1594(1)$ & 7500 & 1 & $136(2)$ \\
\hline $\mathrm{C}(1)$ & $1790(120)$ & 0 & 2500 & 1 & $290(40)$ \\
\hline $\mathrm{H}(1 \mathrm{~A})$ & 1428 & -183 & 3121 & 0.25 & 434 \\
\hline $\mathrm{H}(1 \mathrm{~B})$ & 1428 & -30 & 1487 & 0.25 & 434 \\
\hline $\mathrm{H}(1 \mathrm{C})$ & 1428 & 212 & 2892 & 0.25 & 434 \\
\hline $\mathrm{N}(1)$ & $3360(120)$ & 0 & 2500 & 1 & $290(40)$ \\
\hline $\mathrm{H}(1 \mathrm{D})$ & 3690 & 205 & 2233 & 0.25 & 434 \\
\hline $\mathrm{H}(1 \mathrm{E})$ & 3690 & -154 & 1847 & 0.25 & 434 \\
\hline $\mathrm{H}(1 \mathrm{~F})$ & 3690 & -50 & 3421 & 0.25 & 434 \\
\hline $\mathrm{N}(2)$ & 6852 & 1307 & 7500 & 1 & 199(19) \\
\hline $\mathrm{H}(2 \mathrm{~A})$ & 7342 & 1110 & 7500 & 1 & 299 \\
\hline $\mathrm{H}(2 \mathrm{~B})$ & 6281 & 1320 & 6680 & 0.5 & 299 \\
\hline $\mathrm{H}(2 \mathrm{C})$ & 6281 & 1320 & 8320 & 0.5 & 299 \\
\hline $\mathrm{C}(2)$ & 7934 & 1589 & 7500 & 1 & $200(110)$ \\
\hline$H(2 D)$ & 8567 & 1570 & 6616 & 0.5 & 239 \\
\hline $\mathrm{H}(2 \mathrm{E})$ & 8567 & 1570 & 8384 & 0.5 & 239 \\
\hline $\mathrm{C}(3)$ & 7188 & 1941 & 7500 & 1 & $200(110)$ \\
\hline $\mathrm{H}(3 \mathrm{~A})$ & 6566 & 1967 & 8389 & 0.5 & 239 \\
\hline $\mathrm{H}(3 \mathrm{~B})$ & 6566 & 1967 & 6611 & 0.5 & 239 \\
\hline $\mathrm{C}(4)$ & 8445 & 2208 & 7500 & 1 & $200(110)$ \\
\hline $\mathrm{H}(4 \mathrm{~A})$ & 9067 & 2182 & 8389 & 0.5 & 239 \\
\hline $\mathrm{H}(4 \mathrm{~B})$ & 9067 & 2182 & 6611 & 0.5 & 239 \\
\hline$C(5)$ & 7704 & 2555 & 7500 & 1 & $200(110)$ \\
\hline $\mathrm{H}(5 \mathrm{~A})$ & 8457 & 2728 & 7500 & 1 & 299 \\
\hline $\mathrm{H}(5 \mathrm{~B})$ & 7092 & 2578 & 8385 & 0.5 & 299 \\
\hline $\mathrm{H}(5 \mathrm{C})$ & 7092 & 2578 & 6615 & 0.5 & 299 \\
\hline
\end{tabular}


${ }^{*} U_{\text {eq }}$ is defined as one third of the trace of the orthogonalized $U_{i j}$ tensor.

Table S15. Anisotropic displacement parameters $\left(\AA^{2} \times 10^{3}\right)$ for $(\mathrm{BA})_{2}(\mathrm{MA}) \mathrm{Pb}_{2} \mathrm{I}_{7}(\mathrm{n}=2)$ at $293(2)$

$\mathrm{K}$ with estimated standard deviations in parentheses.

\begin{tabular}{lllllll}
\hline Label & $\mathrm{U}_{11}$ & $\mathrm{U}_{22}$ & $\mathrm{U}_{33}$ & $\mathrm{U}_{12}$ & $\mathrm{U}_{13}$ & $\mathrm{U}_{23}$ \\
\hline $\mathrm{Pb}(1)$ & $44(1)$ & $84(1)$ & $52(1)$ & $-1(1)$ & 0 & 0 \\
$\mathrm{I}(1)$ & $96(2)$ & $74(2)$ & $142(3)$ & 0 & 0 & 0 \\
$\mathrm{I}(2)$ & $89(2)$ & $165(2)$ & $85(2)$ & 0 & $49(2)$ & 0 \\
$\mathrm{I}(3)$ & $81(2)$ & $164(2)$ & $90(2)$ & 0 & $-44(2)$ & 0 \\
$\mathrm{I}(4)$ & $90(2)$ & $75(2)$ & $242(4)$ & $0(2)$ & 0 & 0 \\
\multicolumn{5}{l}{ The anisotropic displacement factor exponent takes the form: $-2 \pi^{2}\left[\mathrm{~h}^{2} \mathrm{a}^{* 2} \mathrm{U}_{11}+\ldots+2 \mathrm{hka}^{*} \mathrm{~b}^{*} \mathrm{U}_{12}\right]}$.
\end{tabular}

Table S16.Selected bond lengths $[\AA]$ for $(\mathrm{BA})_{2}\left(\mathrm{MA}^{2} \mathrm{~Pb}_{2} \mathrm{I}_{7}(\mathrm{n}=2)\right.$ at $293(2) \mathrm{K}$ with estimated standard deviations in parentheses.

\begin{tabular}{cc}
\hline Label & Distances \\
\hline $\mathrm{Pb}(1)-\mathrm{I}(4)$ & $3.081(2)$ \\
$\mathrm{Pb}(1)-\mathrm{I}(3)$ & $3.1644(7)$ \\
$\mathrm{Pb}(1)-\mathrm{I}(2)$ & $3.1721(8)$ \\
$\mathrm{Pb}(1)-\mathrm{I}(1)$ & $3.2633(10)$ \\
\hline
\end{tabular}

Table S17. Selected bond angles $\left[{ }^{\circ}\right]$ for $(\mathrm{BA})_{2}(\mathrm{MA}) \mathrm{Pb}_{2} \mathrm{I}_{7}(\mathrm{n}=2)$ at $293(2) \mathrm{K}$ with estimated standard deviations in parentheses.

\begin{tabular}{cc}
\hline Label & Angles \\
\hline $\mathrm{Pb}(1)^{\prime}-\mathrm{I}(1)-\mathrm{Pb}(1)$ & $165.63(13)$ \\
$\mathrm{Pb}(1)^{\prime}-\mathrm{I}(2)-\mathrm{Pb}(1)$ & $164.54(11)$ \\
$\mathrm{Pb}(1)-\mathrm{I}(3)-\mathrm{Pb}(1)^{\prime}$ & $169.93(11)$ \\
\hline
\end{tabular}


Table S18. Atomic coordinates $\left(\times 10^{4}\right)$ and equivalent isotropic displacement parameters $\left(\AA^{2} \times 10^{3}\right)$ for $(\mathrm{BA})_{2}(\mathrm{MA})_{2} \mathrm{~Pb}_{3} \mathrm{I}_{10}(\mathrm{n}=3)$ at $293(2) \mathrm{K}$ with estimated standard deviations in parentheses.

\begin{tabular}{|c|c|c|c|c|c|}
\hline Label & $\mathrm{x}$ & $\mathrm{y}$ & $\mathrm{z}$ & Occupancy & $\mathrm{U}_{\mathrm{eq}}{ }^{*}$ \\
\hline $\mathrm{Pb}(1)$ & 5000 & 0 & 5000 & 1 & $53(1)$ \\
\hline $\mathrm{Pb}(2)$ & $5013(2)$ & $1239(1)$ & 5000 & 1 & $52(1)$ \\
\hline $\mathrm{I}(1)$ & $4675(4)$ & $604(1)$ & 5000 & 1 & $101(2)$ \\
\hline $\mathrm{I}(2)$ & 7500 & $43(1)$ & 7500 & 1 & $196(3)$ \\
\hline $\mathrm{I}(3)$ & 2500 & $1279(1)$ & 7500 & 1 & $102(2)$ \\
\hline $\mathrm{I}(4)$ & $5586(4)$ & $1817(1)$ & 5000 & 1 & $136(2)$ \\
\hline $\mathrm{I}(5)$ & 7500 & $1156(1)$ & 7500 & 1 & $104(2)$ \\
\hline $\mathrm{N}(1)$ & $540(110)$ & $599(18)$ & 5000 & 1 & $310(50)$ \\
\hline $\mathrm{H}(1 \mathrm{~A})$ & 889 & 746 & 4630 & 0.5 & 459 \\
\hline $\mathrm{H}(1 \mathrm{~B})$ & 853 & 468 & 4431 & 0.5 & 459 \\
\hline $\mathrm{H}(1 \mathrm{C})$ & 867 & 578 & 5938 & 0.5 & 459 \\
\hline$C(1)$ & $-1100(120)$ & $600(20)$ & 5000 & 1 & $310(50)$ \\
\hline $\mathrm{H}(1 \mathrm{D})$ & -1478 & 472 & 5650 & 0.5 & 459 \\
\hline $\mathrm{H}(1 \mathrm{E})$ & -1464 & 577 & 3995 & 0.5 & 459 \\
\hline $\mathrm{H}(1 \mathrm{~F})$ & -1439 & 770 & 5355 & 0.5 & 459 \\
\hline $\mathrm{N}(2)$ & -359 & 1596 & 5000 & 1 & $180(20)$ \\
\hline $\mathrm{H}(2 \mathrm{~A})$ & 142 & 1448 & 5000 & 1 & 269 \\
\hline $\mathrm{H}(2 \mathrm{~B})$ & -933 & 1605 & 4181 & 0.5 & 269 \\
\hline $\mathrm{H}(2 \mathrm{C})$ & -933 & 1605 & 5819 & 0.5 & 269 \\
\hline $\mathrm{C}(2)$ & 756 & 1821 & 5000 & 1 & $180(170)$ \\
\hline $\mathrm{H}(2 \mathrm{D})$ & 1393 & 1802 & 5878 & 0.5 & 215 \\
\hline $\mathrm{H}(2 \mathrm{E})$ & 1393 & 1802 & 4122 & 0.5 & 215 \\
\hline$C(3)$ & 165 & 2097 & 5000 & 1 & $180(170)$ \\
\hline $\mathrm{H}(3 \mathrm{~A})$ & -444 & 2128 & 4113 & 0.5 & 215 \\
\hline $\mathrm{H}(3 \mathrm{~B})$ & -444 & 2128 & 5887 & 0.5 & 215 \\
\hline $\mathrm{C}(4)$ & 1497 & 2273 & 5000 & 1 & $180(170)$ \\
\hline $\mathrm{H}(4 \mathrm{~A})$ & 2107 & 2243 & 4113 & 0.5 & 215 \\
\hline $\mathrm{H}(4 \mathrm{~B})$ & 2107 & 2243 & 5887 & 0.5 & 215 \\
\hline$C(5)$ & 909 & 2550 & 5000 & 1 & $180(170)$ \\
\hline $\mathrm{H}(5 \mathrm{~A})$ & 1738 & 2668 & 5000 & 1 & 269 \\
\hline
\end{tabular}




$\begin{array}{llllll}\mathrm{H}(5 \mathrm{~B}) & 309 & 2578 & 5883 & 0.5 & 269 \\ \mathrm{H}(5 \mathrm{C}) & 309 & 2578 & 4117 & 0.5 & 269\end{array}$

" $U_{\mathrm{eq}}$ is defined as one third of the trace of the orthogonalized $\mathrm{U}_{\mathrm{ij}}$ tensor.

Table S19. Anisotropic displacement parameters $\left(\AA^{2} \times 10^{3}\right)$ for $(\mathrm{BA})_{2}(\mathrm{MA})_{2} \mathrm{~Pb}_{3} \mathrm{I}_{10}(\mathrm{n}=3)$ at 293(2) K with estimated standard deviations in parentheses.

\begin{tabular}{lllllll}
\hline Label & $\mathrm{U}_{11}$ & $\mathrm{U}_{22}$ & $\mathrm{U}_{33}$ & $\mathrm{U}_{12}$ & $\mathrm{U}_{13}$ & $\mathrm{U}_{23}$ \\
\hline $\mathrm{Pb}(1)$ & $45(1)$ & $65(2)$ & $49(1)$ & $-1(1)$ & 0 & 0 \\
$\mathrm{~Pb}(2)$ & $40(1)$ & $68(1)$ & $46(1)$ & $-1(1)$ & 0 & 0 \\
$\mathrm{I}(1)$ & $109(3)$ & $55(2)$ & $138(3)$ & $0(2)$ & 0 & 0 \\
$\mathrm{I}(2)$ & $226(6)$ & $131(4)$ & $231(6)$ & 0 & $-194(5)$ & 0 \\
$\mathrm{I}(3)$ & $72(2)$ & $161(3)$ & $72(2)$ & 0 & $34(2)$ & 0 \\
$\mathrm{I}(4)$ & $94(2)$ & $62(2)$ & $252(6)$ & $0(2)$ & 0 & 0 \\
$\mathrm{I}(5)$ & $77(2)$ & $154(3)$ & $80(2)$ & 0 & $-40(2)$ & 0 \\
\hline
\end{tabular}

The anisotropic displacement factor exponent takes the form: $-2 \pi^{2}\left[h^{2} a^{2}{ }^{2} U_{11}+\ldots+2 h k a b{ }^{*} U_{12}\right]$.

Table S20. Selected bond lengths $[\AA]$ for for $(\mathrm{BA})_{2}(\mathrm{MA})_{2} \mathrm{~Pb}_{3} \mathrm{I}_{10}(\mathrm{n}=3)$ at $293(2) \mathrm{K}$ with estimated standard deviations in parentheses.

\begin{tabular}{cc}
\hline Label & Distances \\
\hline $\mathrm{Pb}(1)-\mathrm{I}(1)$ & $3.154(3)$ \\
$\mathrm{Pb}(1)-\mathrm{I}(2)$ & $3.1555(4)$ \\
$\mathrm{Pb}(2)-\mathrm{I}(4)$ & $3.047(4)$ \\
$\mathrm{Pb}(2)-\mathrm{I}(3)$ & $3.1626(10)$ \\
$\mathrm{Pb}(2)-\mathrm{I}(5)$ & $3.1692(11)$ \\
$\mathrm{Pb}(2)-\mathrm{I}(1)$ & $3.310(3)$ \\
\hline
\end{tabular}

Table S21. Selected bond angles $\left[^{\circ}\right]$ for for $(\mathrm{BA})_{2}(\mathrm{MA})_{2} \mathrm{~Pb}_{3} \mathrm{I}_{10}(\mathrm{n}=3)$ at $293(2) \mathrm{K}$ with estimated standard deviations in parentheses.

\begin{tabular}{cc}
\hline Label & Angles \\
\hline $\mathrm{Pb}(1)-\mathrm{I}(1)-\mathrm{Pb}(2)$ & $169.48(13)$ \\
$\mathrm{Pb}(1)-\mathrm{I}(2)-\mathrm{Pb}(1)$ & $171.89(17)$ \\
$\mathrm{Pb}(2)-\mathrm{I}(3)-\mathrm{Pb}(2)^{\prime}$ & $172.39(15)$ \\
$\mathrm{Pb}(2)^{\prime}-\mathrm{I}(5)-\mathrm{Pb}(2)$ & $164.30(16)$ \\
\hline
\end{tabular}


Table S22. Atomic coordinates $\left(\times 10^{4}\right)$ and equivalent isotropic displacement parameters $\left(\AA^{2} \times 10^{3}\right)$ for $(\mathrm{BA})_{2}(\mathrm{MA})_{3} \mathrm{~Pb}_{4} \mathrm{I}_{13}(\mathrm{n}=4)$ at $293(2) \mathrm{K}$ with estimated standard deviations in parentheses.

\begin{tabular}{|c|c|c|c|c|c|}
\hline Label & $\mathrm{x}$ & $\mathrm{y}$ & $\mathrm{z}$ & Occupancy & $\mathrm{U}_{\mathrm{eq}}^{*}$ \\
\hline $\mathrm{Pb}(1)$ & $7500(1)$ & $493(1)$ & 2500 & 1 & $41(1)$ \\
\hline $\mathrm{Pb}(2)$ & $7489(1)$ & $1484(1)$ & 2500 & 1 & $46(1)$ \\
\hline $\mathrm{I}(1)$ & 5000 & $1416(1)$ & 0 & 1 & $92(1)$ \\
\hline $\mathrm{I}(2)$ & 0 & $1521(1)$ & 0 & 1 & $90(1)$ \\
\hline $\mathrm{I}(3)$ & 10000 & $446(1)$ & 0 & 1 & $112(1)$ \\
\hline $\mathrm{I}(4)$ & $7110(4)$ & 0 & 2500 & 1 & $103(2)$ \\
\hline $\mathrm{I}(5)$ & 7934(3) & $974(1)$ & 2500 & 1 & $102(1)$ \\
\hline $\mathrm{I}(6)$ & 5000 & $532(1)$ & 0 & 1 & $109(1)$ \\
\hline I(7) & $6900(3)$ & 1950(1) & 2500 & 1 & $125(2)$ \\
\hline $\mathrm{C}(1)$ & $1510(90)$ & 0 & 2500 & 1 & $290(40)$ \\
\hline $\mathrm{H}(1 \mathrm{~A})$ & 1150 & -119 & 3045 & 0.25 & 433 \\
\hline $\mathrm{H}(1 \mathrm{~B})$ & 1150 & -6 & 1482 & 0.5 & 433 \\
\hline $\mathrm{H}(1 \mathrm{C})$ & 1150 & 125 & 2973 & 0.25 & 433 \\
\hline $\mathrm{N}(1)$ & $3120(80)$ & 0 & 2500 & 1 & $290(40)$ \\
\hline $\mathrm{H}(1 \mathrm{D})$ & 3453 & 119 & 2109 & 0.25 & 433 \\
\hline $\mathrm{H}(1 \mathrm{E})$ & 3453 & -106 & 1951 & 0.25 & 433 \\
\hline $\mathrm{H}(1 \mathrm{~F})$ & 3453 & -13 & 3440 & 0.5 & 433 \\
\hline$C(2)$ & $3410(70)$ & $983(11)$ & 2500 & 1 & $280(30)$ \\
\hline $\mathrm{H}(2 \mathrm{~A})$ & 3585 & 1114 & 2010 & 0.5 & 419 \\
\hline $\mathrm{H}(2 \mathrm{~B})$ & 3941 & 876 & 1971 & 0.5 & 419 \\
\hline $\mathrm{H}(2 \mathrm{C})$ & 3770 & 990 & 3519 & 0.5 & 419 \\
\hline $\mathrm{N}(2)$ & $1880(60)$ & $939(9)$ & 2500 & 1 & $280(30)$ \\
\hline$H(2 D)$ & 1651 & 863 & 3307 & 0.5 & 419 \\
\hline $\mathrm{H}(2 \mathrm{E})$ & 1643 & 868 & 1671 & 0.5 & 419 \\
\hline $\mathrm{H}(2 \mathrm{~F})$ & 1361 & 1057 & 2522 & 0.5 & 419 \\
\hline $\mathrm{N}(3)$ & 3050 & 1807 & 2500 & 1 & $121(10)$ \\
\hline $\mathrm{H}(3 \mathrm{~A})$ & 2735 & 1675 & 2500 & 1 & 182 \\
\hline $\mathrm{H}(3 \mathrm{~B})$ & 3601 & 1830 & 3318 & 0.5 & 182 \\
\hline $\mathrm{H}(3 \mathrm{C})$ & 3601 & 1830 & 1682 & 0.5 & 182 \\
\hline$C(3)$ & 1745 & 1947 & 2500 & 1 & $100(400)$ \\
\hline
\end{tabular}




\begin{tabular}{llllll}
$\mathrm{H}(3 \mathrm{D})$ & 1135 & 1923 & 3386 & 0.5 & 146 \\
$\mathrm{H}(3 \mathrm{E})$ & 1135 & 1923 & 1614 & 0.5 & 146 \\
$\mathrm{C}(4)$ & 2312 & 2168 & 2500 & 1 & $100(400)$ \\
$\mathrm{H}(4 \mathrm{~A})$ & 2923 & 2192 & 1614 & 0.5 & 146 \\
$\mathrm{H}(4 \mathrm{~B})$ & 2923 & 2192 & 3386 & 0.5 & 146 \\
$\mathrm{C}(5)$ & 953 & 2315 & 2500 & 1 & $100(400)$ \\
$\mathrm{H}(5 \mathrm{~A})$ & 343 & 2289 & 1615 & 0.5 & 146 \\
$\mathrm{H}(5 \mathrm{~B})$ & 343 & 2289 & 3385 & 0.5 & 146 \\
$\mathrm{C}(6)$ & 1486 & 2542 & 2500 & 1 & $100(400)$ \\
$\mathrm{H}(6 \mathrm{~A})$ & 633 & 2633 & 2500 & 1 & 182 \\
$\mathrm{H}(6 \mathrm{~B})$ & 2079 & 2567 & 1617 & 0.5 & 182 \\
$\mathrm{H}(6 \mathrm{C})$ & 2079 & 2567 & 3383 & 0.5 & 182 \\
\hline
\end{tabular}

" $\mathrm{U}_{\text {eq }}$ is defined as one third of the trace of the orthogonalized $\mathrm{U}_{\mathrm{ij}}$ tensor.

Table S23. Anisotropic displacement parameters $\left(\AA^{2} \times 10^{3}\right)$ for $(\mathrm{BA})_{2}(\mathrm{MA})_{3} \mathrm{~Pb}_{4} \mathrm{I}_{13}(\mathrm{n}=4)$ at 293(2) K with estimated standard deviations in parentheses.

\begin{tabular}{lllllll}
\hline Label & $\mathrm{U}_{11}$ & $\mathrm{U}_{22}$ & $\mathrm{U}_{33}$ & $\mathrm{U}_{12}$ & $\mathrm{U}_{13}$ & $\mathrm{U}_{23}$ \\
\hline $\mathrm{Pb}(1)$ & $36(1)$ & $46(1)$ & $42(1)$ & $0(1)$ & 0 & 0 \\
$\mathrm{~Pb}(2)$ & $37(1)$ & $52(1)$ & $48(1)$ & $1(1)$ & 0 & 0 \\
$\mathrm{I}(1)$ & $68(2)$ & $137(2)$ & $70(2)$ & 0 & $-34(1)$ & 0 \\
$\mathrm{I}(2)$ & $64(2)$ & $139(2)$ & $67(2)$ & 0 & $31(1)$ & 0 \\
$\mathrm{I}(3)$ & $92(2)$ & $141(2)$ & $102(2)$ & 0 & $64(2)$ & 0 \\
$\mathrm{I}(4)$ & $103(2)$ & $36(2)$ & $171(4)$ & 0 & 0 & 0 \\
$\mathrm{I}(5)$ & $98(2)$ & $39(1)$ & $167(3)$ & $-2(1)$ & 0 & 0 \\
$\mathrm{I}(6)$ & $91(2)$ & $144(2)$ & $91(2)$ & 0 & $-63(2)$ & 0 \\
$\mathrm{I}(7)$ & $76(2)$ & $48(1)$ & $253(4)$ & $1(1)$ & 0 & 0 \\
\hline The anisotropic displacement factor exponent takes the form: $-2 \pi^{2}\left[\mathrm{~h}^{2} \mathrm{a}^{2}{ }^{2} \mathrm{U}_{11}+\ldots+2 \mathrm{hka} \mathrm{b}^{*} \mathrm{U}_{12}\right]$.
\end{tabular}


Table S24. Selected bond lengths $[\AA]$ for $(B A)_{2}(M A)_{3} \mathrm{~Pb}_{4} \mathrm{I}_{13}(\mathrm{n}=4)$ at $293(2) \mathrm{K}$ with estimated standard deviations in parentheses.

\begin{tabular}{cc}
\hline Label & Distances \\
\hline $\mathrm{Pb}(1)-\mathrm{I}(5)$ & $3.1239(18)$ \\
$\mathrm{Pb}(1)-\mathrm{I}(6)$ & $3.1579(6)$ \\
$\mathrm{Pb}(1)-\mathrm{I}(3)$ & $3.1629(6)$ \\
$\mathrm{Pb}(1)-\mathrm{I}(4)$ & $3.1916(8)$ \\
$\mathrm{Pb}(2)-\mathrm{I}(7)$ & $3.044(2)$ \\
$\mathrm{Pb}(2)-\mathrm{I}(2)$, & $3.1639(6)$ \\
$\mathrm{Pb}(2)-\mathrm{I}(1)$ & $3.1725(7)$ \\
$\mathrm{Pb}(2)-\mathrm{I}(5)$ & $3.3091(18)$ \\
\hline
\end{tabular}

Table S25. Selected bond ond angles $\left[^{\circ}\right]$ for $(B A)_{2}(M A)_{3} \mathrm{~Pb}_{4} \mathrm{I}_{13}(\mathrm{n}=4)$ at 293(2) K with estimated standard deviations in parentheses.

\begin{tabular}{cc}
\hline Label & Angles \\
\hline $\mathrm{Pb}(2)-\mathrm{I}(1)-\mathrm{Pb}(2)^{\prime}$ & $163.92(9)$ \\
$\mathrm{Pb}(2)^{\prime}-\mathrm{I}(2)-\mathrm{Pb}(2)^{\prime}$ & $171.45(9)$ \\
$\mathrm{Pb}(1)^{\prime}-\mathrm{I}(3)-\mathrm{Pb}(1)$ & $169.05(10)$ \\
$\mathrm{Pb}(1)^{\prime}-\mathrm{I}(4)-\mathrm{Pb}(1)$ & $167.48(12)$ \\
$\mathrm{Pb}(1)-\mathrm{I}(5)-\mathrm{Pb}(2)$ & $165.97(10)$ \\
$\mathrm{Pb}(1)-\mathrm{I}(6)-\mathrm{Pb}(1)^{\prime}$ & $170.89(10)$ \\
\hline
\end{tabular}

